\title{
Constructing Unimaginable Subjects: New Economies of Architecture, Design, and Ecology
}

\author{
FILIP TEJCHMAN \\ University of Wisconsin-Milwaukee
}

\begin{abstract}
Constructing Unimaginable Subjects presents studio work that examines the ecological liabilities associated with an architecture-as-service model of practice.
\end{abstract}

In the opening chapter of The Great Derangement, the writer, Amitav Ghosh, muses that the challenges and imminent crisis related to global climate change are "...a crisis of culture, and thus of the imagination." Architectural language and design culture could be included in this crisis by association. Perhaps the more immediate crisis - for architects - is the glut of assumptions and accompanying sure-footedness with which the discipline and profession offer "solutions." To some extent, this is symptomatic of a commonly accepted over-simplification of the mechanisms and flows of the environment, in which weather, flora, fauna, economies, cities, cultures, and people, are cast as easily distinguishable nodes. Within this schema, cause and effect are guided along a linear path wherein climate change is the culminating outcome, while simultaneously providing a universal measure for transformation in every aspect and scale of the observable world.

A more lucid mapping would reveal that climate change is an aggregate of multiple symptoms, multiple pathways that encode the effects of change onto ecologies, and viceversa. Despite the global proliferation and recognition of a diverse range of consequences attributed to climate change, the conception of architectural agency in response - or as a potential response - remains bound to a 20th-century concept of design. This means that while the discipline and profession of architecture may desire to project their influence, the methods deployed are generally limited to modalities of design that can be grouped into categories best described as either thermodynamic plasticity, the search for optimized geometries, or the more familiar and pervasive, green-washing of the status quo vis a vis the protocols of sustainability and resilience. This is reflected in the legacy of "environmental architecture," which is almost universally concerned with questions of building performance generally expressed as either form and/or material response to context, or an investigation/application of novel environmental control systems. Though examples of wellregarded and critical work can be found in either category, a rarely discussed aspect is just how limited an impact these buildings can make, after all it is a well documented fact that Architects design only a small percentage of the built world. Whether intentional or inevitable, the discipline has found itself in an awkward situation; an outspoken critic of the negligent habits of the AEC (Architecture Engineering Construction) industry at-large, while remaining committed to the production of bespoke luxury objects. However, even the tacit acknowledgement of the latter fails to reveal the external pressures that both the profession and academia are regularly subject to, and which are increasingly difficult to address through existing traditions of design and project delivery.

More concerning is that these pressures are largely unacknowledged, or mistakenly disassociated from on another. A draft report, "Governance of Economic Transition" by the BIOS Research Group, produced as part of the United Nations 2019 Global Sustainable Development Report, revealed the reciprocity between the decline of ecosystems and an incremental economic down-turn. It may seem perverse to discuss the broader health of the global economy during a period of exuberant growth, especially after the near collapse of financial markets ten years ago, but the trajectory of change is gradual and extends forward and backward in time. The BIOS report focuses on several co-extensive long-term trends. First is that the extraction of fossil fuels has become progressively more energy intensive, requiring more effort, and with diminishing yields. Second, economies have reached the capacity of ecosystems to absorb the waste flows associated with the production of energy and materials. These negative externalities, or "sink" costs, are both local and global in impact. Local effects like air and water pollution, for instance, exert immediate tolls on health and well-being. These rarely consider the soft-costs associated with adapting to living within a degraded environment, such as the use of air and water filtration systems, or an increase in the need for artificial lighting and ventilation. When combined with the slow but steady decrease in the efficiency of energy/material production, as well as the planet's sink capacity, the asymmetric ways in which the hidden costs of climate change are distributed will radically alter the institutions, structures, and subject of architecture in the near-future.

While these material, economic, and energetic ecologies are simultaneously complicit in the production of this crisis, they are also the primary lens through which we can mediate and understand the relationship of architecture to the unimaginable. Thus the shared challenge to humanity, can also be parsed in very specific disciplinary and cultural terms. Specifically, since the industrial revolution the core occupation of architects has largely favored a service model underwritten by 
either governments or patrons/clients. It is impossible to recognize every permutation of architecture-as-service intrinsic to the institutions, norms, habits, and pedagogies of the profession. Nevertheless, this bias persists and is manifested in countless benign actions and situations throughout practice and academia. With regard to understanding and proposing productive architectural engagements with ecologies, this unspoken bias towards service intercedes in a way that unconsciously compels us to solely conceive of the problem within the accepted tropes of design.

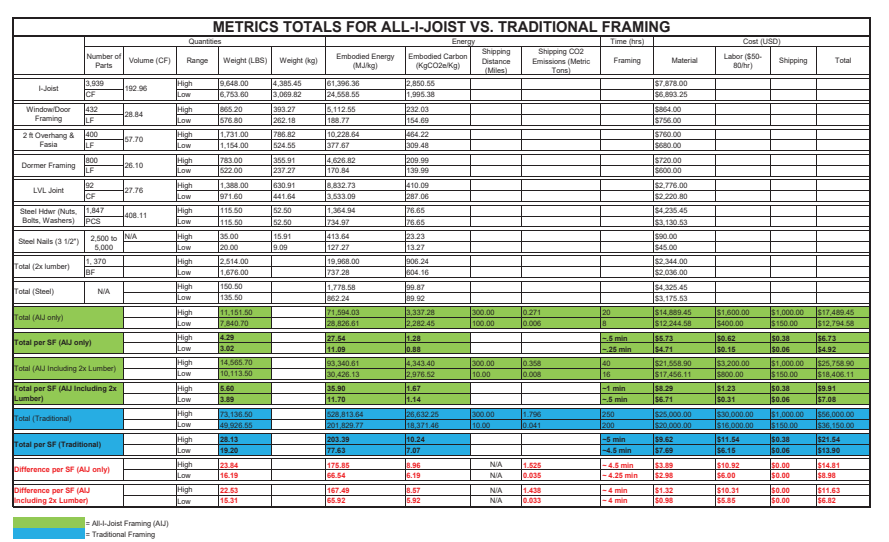

It is within this context of converging crisis that the Components Elements Parts Bits (CEPB) studio was conceived. Taught in collaboration by faculty from the Schools of Architecture, Engineering, and Business, the underlying premise was to challenge the primacy of architecture-as-service in order to reconsider architecture as a product. Shifting the focus away from normative modes of practice had a reciprocal effect on the corresponding design conversation. In particular, the examination of how certain material and economic adjacencies are easily misinterpreted as indicators

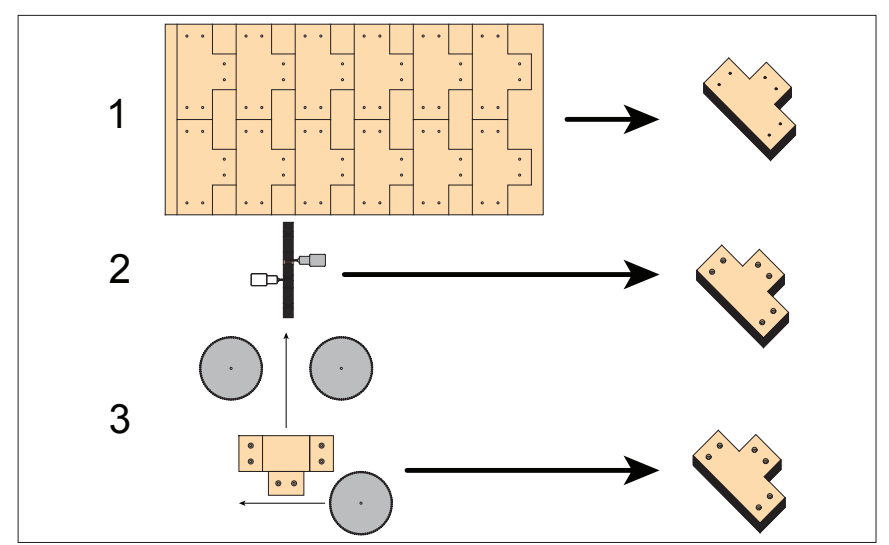

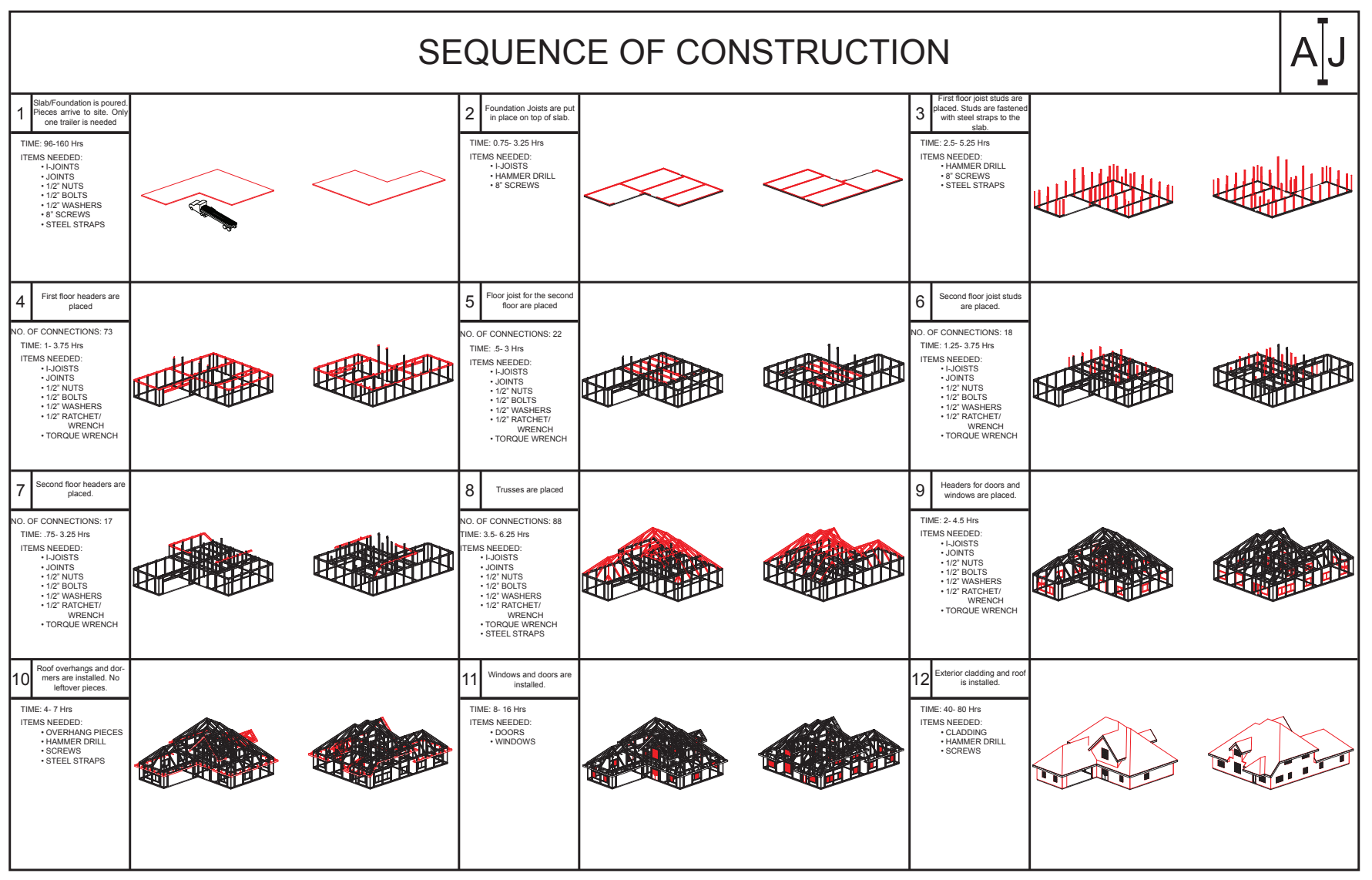

Figure 1. "All I-Joist System" Student(s):Jack Jenkins and Daniel Morales. 
of broader cultural desires. For example, the varying average square footage of a residential home, which is determined by labor, material, and development costs, is not a direct indicator of market preference. Architecture's environmental costs were initially adopted as the guiding metric, though this presented some unforeseen challenges. While residential and commercial building operations consume approximately $40 \%$ (yearly) of the energy produced in North America, this figure does not accurately represent the full scope of architectures environmental entanglement. In addition to the fuel(s) converted into heating, cooling, lighting, and movement of various building mechanisms, if we added the related environmental costs of resource extraction, processing, shipping of materials, and the logistics of site work involved in the process of construction, we would arrive at what would seem to be a more comprehensive quanta. Hidden within the most rigorous and totalizing examination of embodied energy within buildings is a record of the dynamics of environmental change reflexively encoded within the decision making processes. For example, how do environmental sink costs alter
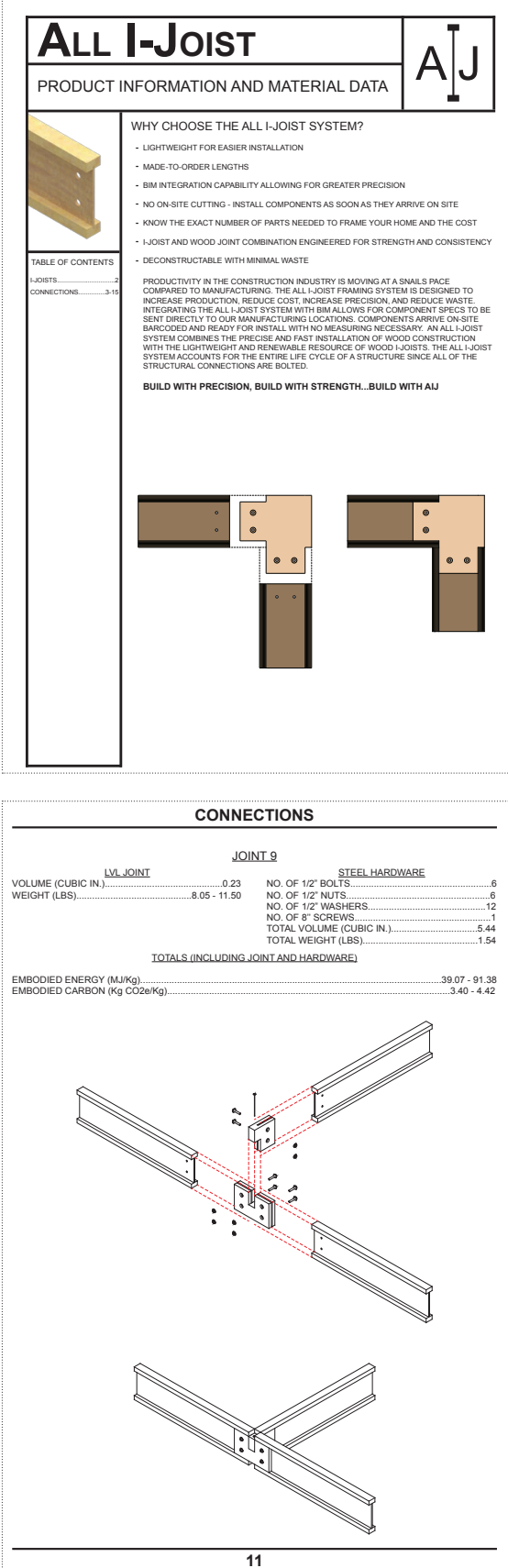
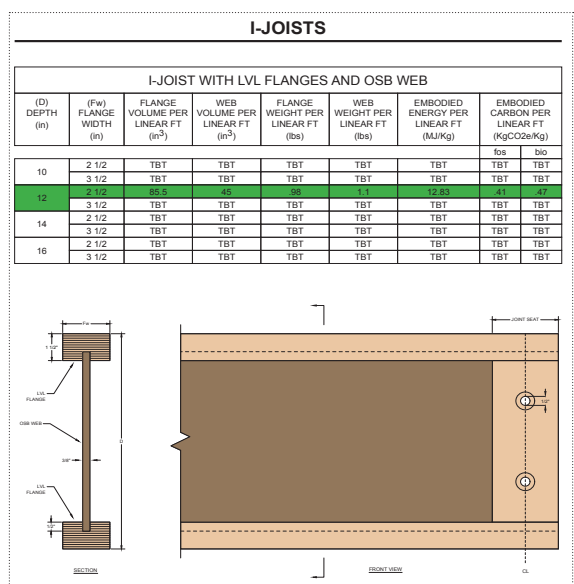

$\underline{\operatorname{scons}}$
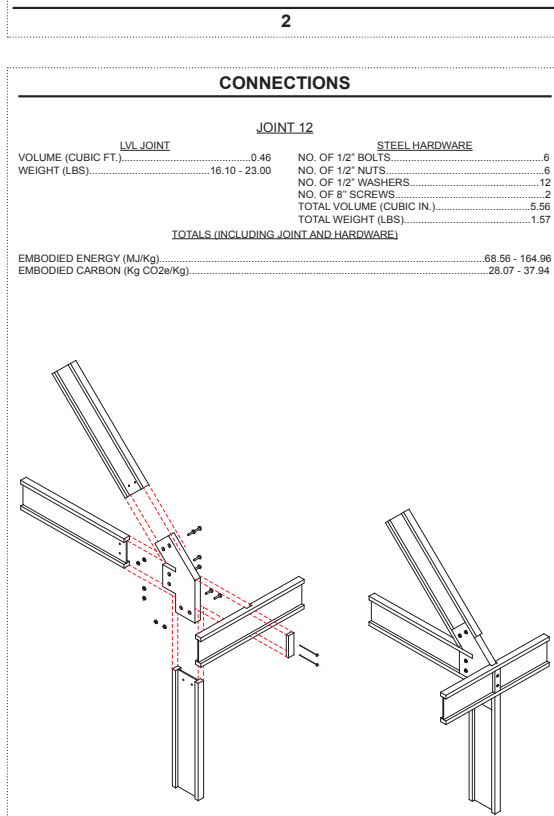

14
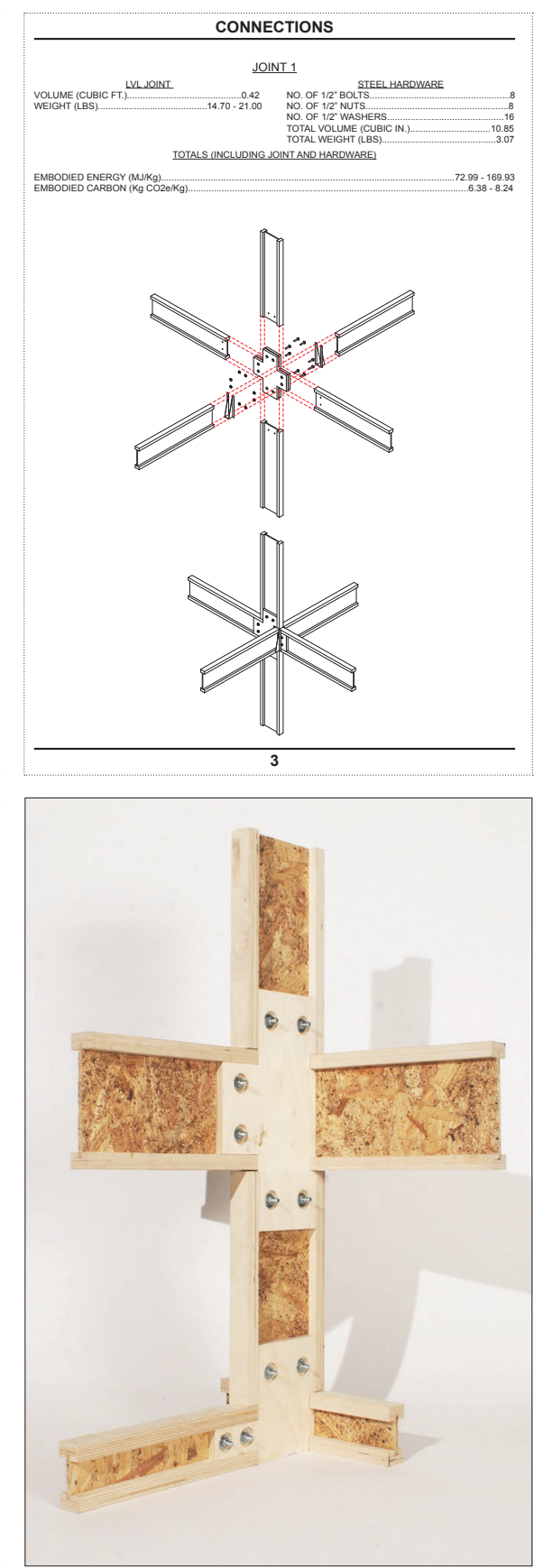

Figure 2. "All I-Joist System" Student(s):Jack Jenkins and Daniel Morales. 
labor and material costs? Or, what factors will have exert the greatest burden during construction? The embodied energy costs of an intricate light-weight structure might look good on paper, but these do not reflect the costs that are expended on the supporting labor and equipment. "How much does your building weigh?" can under varying circumstances be outmoded by examining the ease with which it is recycled/ composted, or adapted for another use.

The CEPB studio addressed the limits of contemporary design practice in response to environmental challenges by shifting the emphasis from how a building is configured, towards how an ecological network of parts, materials, and information can be configured for building. Students were first tasked with identifying as many steps and processes in the respective supply-chains of commonly utilized building materials and assemblies - a "farm-to-table" type of mapping that attempted to consider as many interactions as possible. These analysis were then "audited" based on their carbon emissions and embodied energy. This stage of project research was followed by two distinct field-work models: Customer-Discovery and Field Trips.

Field work played a critical role in the CEPB studio's attempt to un-make the architecture-as-service model because it revealed - on a case-by-case basis - the various presumptions that we necessarily leverage in order to make a proposal, such as the constraints of site or program, for instance. Moreover field work provided counter-examples and references from which a new critical language could be developed. This meant that projects were critiqued based on the outcomes of their respective research rather than the familiar paradigms common to design studios. The choice and use of representational method was contingent on the specific needs of the market/customer. If the product-market fit identified by the student focused on sub-contractors or distributers, for example, then renderings were eschewed in favor of pricing/time-saving spread-sheets, typical details, and assembly diagrams.

Field trips to various factories within the mid-mid-western region focused on industries that specialized in the architectural-scale production of non-architectural things - notable among these were the shipyards of Fincantieri Marinette Marine and Trek Bicycles, as well as the research and development facilities of the USDA Forest Products Research Laboratory. Of acute interest to the studio were the techniques, tools, and methods that other industries utilize to manage the delivery and application of information within a space of production, as well as to optimize material and waste flows.

If Architecture is to seriously consider a shift from service to product, than a number of familiar subjects and constraints will need to be reconsidered. Site, client, and program, for example, are intrinsically biased toward the production of a very specific concept of solution. In the context of the CEPB studio, the latter were deliberately absent so as to remain ambiguous. Instead, an entrepreneurial method similar to Lean LaunchPad was adopted. The latter is premised on the use of customer interviews as a means to quickly verify a hypothesis about a potential business model. What differentiates this approach from more traditional business planning methods is that the research being done is focused on identifying a customer need rather than looking for a product-market fit. In this case, students interviewed contractors, distributors, product wholesalers and manufacturers, as well as architects, developers, and mortgage lenders, in order to more fully understand the needs of the market. In one particular example which was later further refined through an NSF Regional I-Corps grant, a student proposed a light-weight wood "brick" system that would allow anyone to build their own home. As an architectural project, it appeared to satisfy a constellation of typical concerns. During the customer discovery and interview phase, however, it became clear that a "pivot" or adjustment was necessary. Among the key factors was the way that construction loans are administered by lenders, which severely limits the funds available to those that wish to do-it-themselves. Another valuable reveal was the complaints raised by several general contractors and sub-contractors, about the shortage of skilled labor. Thus it became evident that the DIY'er was not the target customer, but that instead professional builders would benefit from the use of a system that allowed them to use more unskilled labor.

The ecological implications of an architecture as product model may seem nebulous given that many of the studio project examples appear to respond exclusively to market demands. However, the promise of this approach is in recognizing that many decisions with profound impacts on the environment are primarily economic. Underlying many of the projects were the conclusions of the recently published McKinsey Global Institute Report: "Reinventing construction through a productivity revolution," which highlights that productivity in construction has increased by less than $1 \%$ in almost 20 yrs! Though efficiency and productivity in building are not directly correlated to the their environmental costs, this statistic does suggest that the $A E C$ industry is likely not currently equipped to accurately track its ecological impact, let alone manage it.

Perhaps the deeper lesson is recognizing that the territories of buildings and ecologies are part of a dynamic that is influenced by economic forces in ways that traditional design cultures either avoid or deploy as low-hanging critical rhetoric. Whether architectural practice shifts towards a more product-centric approach in the future, or continues as the provider of a service is offered here primarily as a point of departure...an opportunity to re-imagine the institution of practice as a means to anticipate and respond to the unimaginable transformation of the ecosystem. 


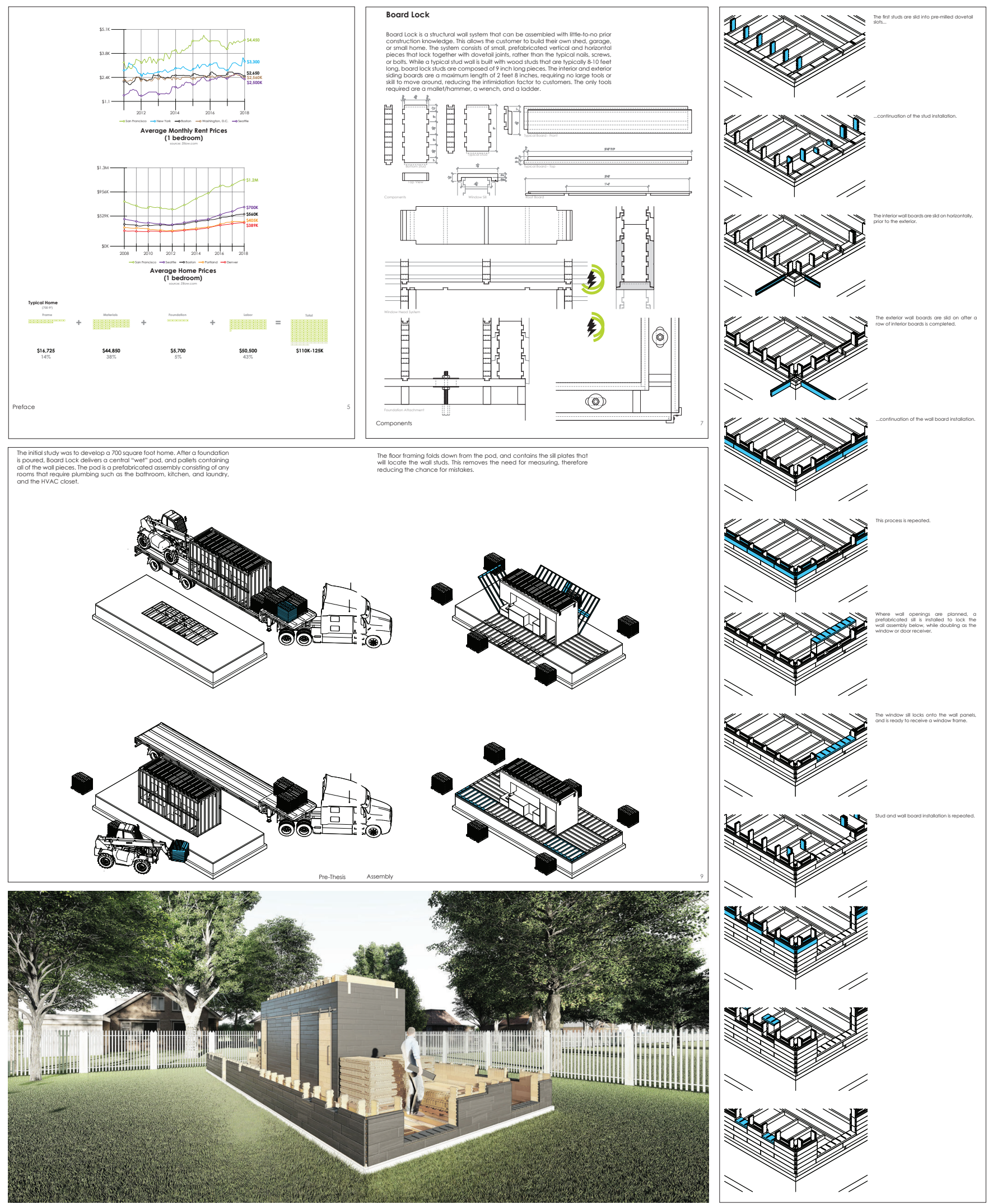



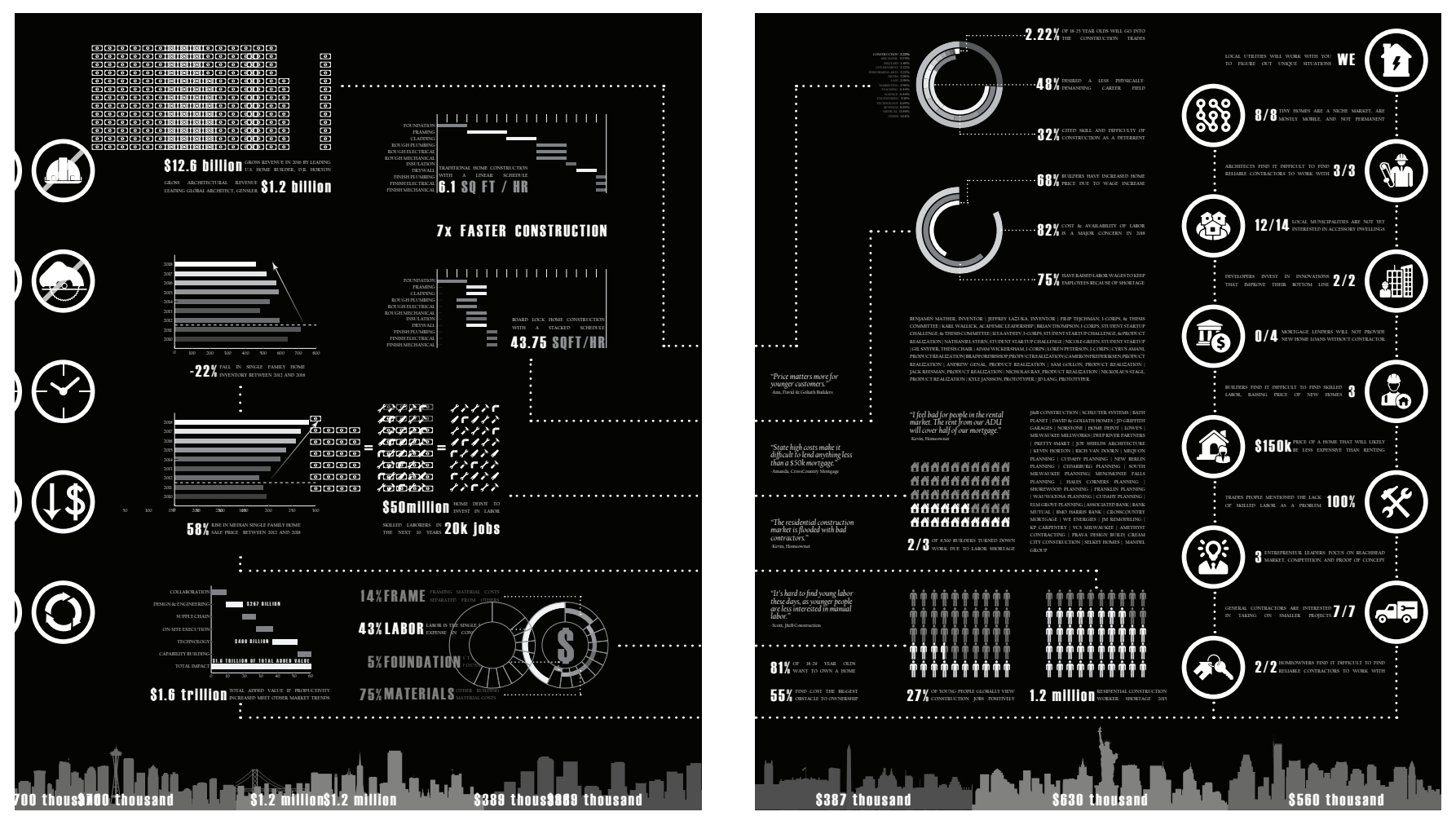

Figure 4. "Boardlock" Student: Ben Mather.

\section{ENDNOTES}

1 Amitav Ghosh, The Great Derangement: Climate Change and the Unthinkable (Chicago: University of Chicago Press: 2017)

2 Paavo Järvensivu, et al., "Governance in Transition" in Global Sustainable Development Report 2019, August 14, 2018. https://bios.fi/bios-governance_of_ economic_transition.pdf

3 This figure is from the United States Department of Energy: energy.gov.

4 Filipe Barbosa, et al., "Reinventing Construction: A Route to Higher Productivity," McKinsey Global Institute Report, February 2017 